\title{
La dyskératose congénitale
}

\section{Une maladie méconnue due à un maintien défectueux des télomères}

> La dyskératose congénitale (DC), ou syndrome de Zinsser-Cole-Engman, est une maladie héréditaire, trop souvent létale, décrite pour la première fois sur le plan dermatologique par Zinsser en 1906. II s'agit d'une maladie très polymorphe sur le plan clinique, variable dans son mode de transmission et dont certains symptômes cliniques apparaissent tardivement, rendant son diagnostic clinique difficile. À cette hétérogénéité clinique est associée une hétérogénéité génétique: on sait que plusieurs gènes, quatre étant identifiés à ce jour, sont impliqués; néanmoins, pour la majorité des patients, le gène en cause n'est pas connu. Le point remarquable est que les quatre gènes connus ( $D K C 1, h T E R C$, hTERT et NOP10) codent des composants de la télomérase tous impliqués dans le maintien de la longueur des télomères, faisant de la DC un modèle clinique unique pour la compréhension du rôle et de l'importance de la télomérase et des télomères. En outre, les protéines codées par les gènes $D K C l$ et NOPIO sont également des composants des particules dites H/ACA impliquées dans la synthèse des ribosomes et des ARN messagers matures. Des altérations de ces deux processus pourraient contribuer aux symptômes des patients atteints de $D C$ due à des mutations de DKCI ou NOP10. <

\section{Le phénotype clinique}

Le phénotype de la dyskératose congénitale (DC), ou syndrome de Zinsser-Cole-Engman [1], est très variable d'un patient à l'autre et, pour un patient donné, évolutif au cours du temps. La description précise du phénotype a été grandement facilitée par la mise en place en 1995 par l'équipe d'Inderjet Dokal à Londres d'un registre international de la DC [2]. Ce registre associe des données cliniques détaillées et des prélèvements sanguins pour les membres malades ou asymptomatiques de 270 familles.

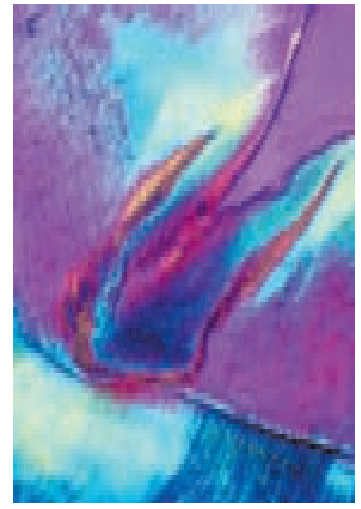

- La forme typique de la $D C$ est caractérisée par des atteintes cutanées, phanériennes et muqueuses: aspect C. Hoareau-Aveilla, Y. Henry : Laboratoire de Biologie Moléculaire Eucaryote du CNRS, Université de Toulouse, UPS, 118 route de Narbonne, 31062 Toulouse cedex 09, France. T. Leblanc: Service de Pédiatrie à orientation hématologique et Centre de Référence des aplasies médullaires constitutionnelles rares, Hôpital Saint-Louis, 1, avenue Claude Vellefaux, 75475 Paris Cedex 10, France. henry@ibcg.biotoul.fr thierry.leblanc@sls.aphp.fr réticulé et hyperpigmentation (mélanodermie) de la peau associé à des zones d'atrophie, dystrophie des ongles (présence de stries, de déformations, réduction de taille voire absence totale des ongles) et leucoplasies des muqueuses, en particulier au niveau de la cavité buccale [3-6]. Les signes cutanés apparaissent en général avant l'âge de 10 ans.

- Les patients peuvent aussi développer un retard statural, une perte précoce de cheveux ou l'apparition prématurée de cheveux blancs, un larmoiement anormal (épiphora), des anomalies dentaires, une ostéoporose précoce, une cirrhose du foie, des sténoses de l'œsophage, des atteintes neurologiques (retard de développement, microcéphalie, atteinte cérébelleuse), une fibrose pulmonaire et un déficit immunitaire global [3, 6] (Tableau I).

- Au moins $80 \%$ des patients développeront une pancytopénie secondaire à une aplasie médullaire. Cette aplasie médullaire apparaît le plus souvent lors de la seconde décade de la vie.

- Enfin, près de $10 \%$ des patients seront atteints de cancers - principalement cancers de la peau, des muqueuses, de l'œsophage - ou d'hémopathies malignes, leucémies ou lymphomes. 
- Les principales causes des décès précoces sont l'insuffisance médullaire, le déficit immunitaire, la fibrose pulmonaire et les néoplasies [7].

Des formes particulières de DC sont également décrites:

- Le syndrome de Hoyeraal-Hreidarsson (HH) a été décrit pour la première fois par Hoyeraal en 1970 [8]. On sait actuellement qu'il s'agit d'une forme très sévère de DC. Il est défini par la présence de quatre critères cliniques parmi les six suivants : retard de croissance intra-utérin (RCIU), retard psychomoteur, microcéphalie, hypoplasie du cervelet, aplasie médullaire précoce et déficit immunitaire sévère. Ces symptômes apparaissent chez le petit enfant. La transmission peut être soit liée au sexe, soit autosomique récessive (Tableau II).

- On a rapporté, chez certains patients ne présentant pas les atteintes cutanées typiques de la DC et chez lesquels le diagnostic d'aplasie médullaire ou de fibrose pulmonaire idiopathique a été posé, des mutations dans les gènes hTERC ou hTERT (cf. infra), également affectés dans les formes plus classiques de la DC [7, 9-14].

\section{Le phénotype cellulaire}

Le retentissement de la $D C$ est maximum pour les tissus nécessitant un renouvellement cellulaire rapide et constant (peau, muqueuses,

\begin{tabular}{|l|l|}
\hline Symptômes & $\begin{array}{c}\text { Pourcentage des } \\
\text { patients affectés }\end{array}$ \\
\hline Atteintes cutanées & $89 \%$ \\
\hline Dystrophie des ongles & $88 \%$ \\
\hline Aplasie médullaire & $85 \%$ \\
\hline Leucokératose & $78 \%$ \\
\hline Larmoiement anormal & $30 \%$ \\
\hline Atteintes neurologiques & $25 \%$ \\
\hline (retard de développement) & $20 \%$ \\
\hline Maladies pulmonaires & $19,5 \%$ \\
\hline Retard statural & $17 \%$ \\
\hline Anomalies dentaires & $17 \%$ \\
\hline Sténoses de l'œsophage & $16 \%$ \\
\hline Perte de cheveux/cheveux blancs prématurés & $8 \%$ \\
\hline Cancers & $7 \%$ \\
\hline Cirrhose du foie & $6 \%$ \\
\hline Microcéphalie & $5 \%$ \\
\hline Ostéoporose précoce & \\
\hline
\end{tabular}

Tableau I. Fréquence de différents symptômes chez des patients atteints de DC (d'après [3]). système digestif, système hématopoïétique). Chez les patients atteints de DC, les cellules de ces tissus présentent deux caractéristiques: (1) elles ont une propension à produire des réarrangements chromosomiques $[4,15]$; et (2) les télomères (structures particulières présentes aux extrémités des chromosomes) sont plus courts que ceux de cellules identiques d'individus sains d'âge équivalent [16]. En ce qui concerne le système hématopoïétique, on note une réduction du nombre de précurseurs hématopoïétiques.

\section{Modes de transmission et anomalies génétiques responsables de la maladie}

La DC est une maladie rare puisqu'on estime sa prévalence à une personne sur un million. Trois modes distincts de transmission de la maladie ont été identifiés [17] (Tableau II) : transmission liée au sexe (MIM Mendelian Inheritance in Man- $\mathrm{n}^{\circ} 305000$ ), transmission autosomique dominante (MIM 127550 ), et transmission autosomique récessive (MIM 224230 ).

\section{La forme liée au chromosome $X$}

est due à des mutations dans le gène codant la protéine dyskérine

La forme la plus classique, qui est aussi la plus sévère, est de transmission liée au sexe. L'étude d'une famille très nombreuse dont seuls les hommes étaient atteints a permis de localiser en 1986 le gène muté responsable de la maladie à l'une des extrémités du chromosome $X$ (locus Xq28) [18]. II a fallu attendre 1998 pour que le gène responsable, $D K C 1$, codant la protéine dyskérine, soit identifié [19]. Les patients atteints de DC pour lesquels on identifie une mutation de $D K C 1$ représentent $31 \%$ des cas [20].

La protéine dyskérine est une enzyme, capable de catalyser la conversion d'uridines en pseudo-uridines (Figure 1A), un dérivé très proche, qui permet une stabilisation des molécules d'ARN [21, 22]. La protéine dyskérine n'agit jamais seule mais est toujours associée à trois protéines, nommées Garl, Nhp2 et Nop10, et à une petite molécule d'ARN contenant des motifs caractéristiques désignés boîte $H$ et boîte ACA [23, 24] (Figure IB). II existe des centaines de petits ARN à boîtes $H$ et $A C A$, auxquels s'associent les protéines dyskérine, Garl, Nhp2 et Nop10, pour former autant de particules dites ribonucléoprotéiques ou RNP (chaque particule contient les quatre protéines et un seul petit ARN H/ACA). Selon la nature exacte du composant ARN qu'elles contiennent, ces particules jouent des rôles divers [25] (Figure 2): 


\begin{tabular}{lcccc}
\hline & DKC1 & TERC & $?$ & Total \\
\hline Transmission liée à I'X & $21(4)$ & $0(0)$ & $1(1)$ & $22(5)$ \\
\hline 2 sujets masculins atteints & $11(3)$ & $1(0)$ & $7(1)$ & $19(4)$ \\
\hline l sujet masculin atteint & $40(8)$ & $3(0)$ & $80(15)$ & $123(23)$ \\
\hline Transmission AD & 0 & $7(0)$ & $4(0)$ & $11(0)$ \\
\hline Transmission AR & 0 & 0 & $18(9)$ & $18(9)$ \\
\hline Cas sporadique & 0 & 0 & $35(7)$ & $35(7)$ \\
\hline Total & 72 & 11 & 145 & 228 \\
\hline
\end{tabular}

Tableau II. Incidence des mutations identifiées chez les patients en fonction du mode de transmission établi cliniquement par l'étude familiale. Sont indiqués le nombre de cas et, entre parenthèses, le nombre de cas dont la présentation clinique est celle d'un syndrome de Hoyeraal-Hreidarsson. Dans cette étude, aucune mutation n'était identifiée chez $63 \%$ des patients (d'après [20]). NBl : les mutations du gène TERT n'avaient pas été recherchées chez l'ensemble des patients et ne figurent pas dans ce tableau (voir texte de l'article [20]). NB2 : les mutations du gène TINF2, rapportées en 2007, ne figurent pas non plus dans cette étude ; le mode de transmission est autosomique dominant et des mutations de ce gène pourraient concerner une partie notable des patients de ce groupe : dans la seule étude rapportée à ce jour, elles sont en effet plus fréquentes que les mutations du gène TERC.

- Certaines RNP H/ACA sont requises lors de la synthèse des ribosomes pendant laquelle elles vont soit introduire des pseudo-uridines au sein des ARN ribosomiques, soit participer à l'extraction, à partir d'une molécule précurseur, des ARN ribosomiques matures [26].

- D'autres RNP H/ACA introduisent des pseudo-uridines au sein des petits ARN du complexe d'épissage, qui joue un rôle crucial dans l'expression des gènes [27]. Toute cellule procède à la synthèse de répliques éphémères des gènes, constituées d'ARN et dénommées pré-ARN messagers. Ces pré-ARN messagers contiennent des régions internes qui les rendent inactifs. Ces régions doivent être excisées, par le complexe d'épissage, pour produire des ARN messagers matures qui pourront être lus par les ribosomes pour produire les protéines correspondantes. L'activité du complexe d'épissage est absolument tributaire de l'introduction par des RNP H/ACA de pseudo-uridines dans certains petits ARN du complexe [28].

- Enfin, la famille des RNP H/ACA comporte un membre atypique: la télomérase de vertébrés [29-31]. La télomérase permet l'ajout aux extrémités des chromosomes, les télomères, de séquences répétées lors de la duplication des chromosomes précédant la division celIulaire $[32,33]$. Les télomères participent de manière cruciale à la sauvegarde de l'intégrité des chromosomes en empêchant par exemple des fusions de chromosomes. Les cellules somatiques saines (toutes les cellules d'un individu à l'exception des cellules produisant les gamètes et les gamètes eux-mêmes) expriment de manière transitoire une forme active de la télomérase à des taux variables selon les types cellulaires [34]. Cette expression transitoire de la télomérase n'est pas suffisante pour empêcher le raccourcissement des télomères à chaque division cellulaire. Néanmoins, elle est essentielle pour assurer le potentiel prolifératif des cellules. Par exemple, l'inhibition de la télomérase dans des fibroblastes en culture ralentit leur prolifération et induit leur entrée prématurée dans un état quiescent, non-prolifératif, dit de «sénescence réplicative 》 $[34$, $60,62,63](\rightarrow)$. Les cellules souches à $(\rightarrow)$ voir l'article de Eric Gilson et al., p. 383 de ce numéro l'origine du renouvellement des cellules sanguines et de tissus comme la peau et les muqueuses, doivent avoir une capacité proliférative élevée, certainement tributaire de l'expression d'une forme active de la télomérase. L'activité catalytique proprement dite de la télomérase est apportée par une protéine à activité transcriptase inverse (nommée hTERT chez l'humain) qui se sert de la région dite matrice du composant ARN de la télomérase (ARN nommé hTERC chez l'humain) pour synthétiser des répétitions télomériques. L'ARN de la télomérase (voir Figure 3) contient en outre un domaine apparenté aux ARN H/ACA classiques, qui comporte les boîtes H et ACA caractéristiques et qui s'associe aux protéines dyskérine, Garl, Nhp2 et Nop10 [29-31, 35]. La partie de la télomérase constituée du domaine H/ACA de l'ARN associé à la dyskérine, Garl, Nhp2 et Nop10 joue un rôle essentiel dans la stabilisation du complexe télomérase et dans sa localisation correcte au sein du noyau des cellules.

Dans tous les cas décrits ci-dessus, la protéine dyskérine joue un rôle crucial lors de l'assemblage des particules RNP H/ACA et dans le maintien de leur intégrité. Ces rôles découlent de la capacité supposée de la dyskérine à se fixer directement sur les ARN H/ACA (ce qui a été démontré sans ambiguité dans le cas de l'équivalent de la dyskérine chez les archées, nommé Cbf5 [36, 37]). Elle est aussi directement responsable de l'activité de peudo-uridylation des particules qui ont cette fonction. La dyskérine est une protéine modulaire, composée de domaines ayant des rôles distincts (Figure 4). Le domaine PUA de la dyskérine fixe directement certaines tiges des ARN H/ACA, alors que le domaine TRUB est le domaine catalytique en tant que tel qui interagit le cas échéant avec l'ARN substrat pour catalyser la conversion d'uridines en pseudo-uridines.

Les mutations du gène codant la dyskérine responsables de la $D C$ sont groupées pour l'essentiel dans la séquence du gène codant le domaine PUA et dans une séquence codant un domaine de fonction mal définie en amont du domaine TruB (Figure 4). II s'agit presque toujours de 
mutations qui aboutissent à la substitution d'un acide aminé [20]. L'étude du phénotype clinique en fonction des mutations de DKCl n'a pas identifié de corrélation évidente entre le type de mutation observée et le phénotype clinique. Cette absence de corrélation est illustrée de manière éloquente par la découverte que certains patients atteints du syndrome de $\mathrm{HH}$ avaient aussi des mutations de DKC1 [38-40] et qu'une des mutations de DKCl identifiée chez un patient atteint du syndrome de $\mathrm{HH}$ était également responsable chez d'autres patients d'une forme beaucoup plus classique de la DC [5]. Les facteurs génétiques ou environnementaux qui modulent l'impact des mutations de $D K C l$ restent pour le moment inconnus.

La forme de transmission autosomique dominante peut être due à des mutations dans le gène hTERC ou le gène hTERT

En 2001, il a été démontré que dans plusieurs familles, la forme autosomique dominante de la DC était due à des mutations dans le

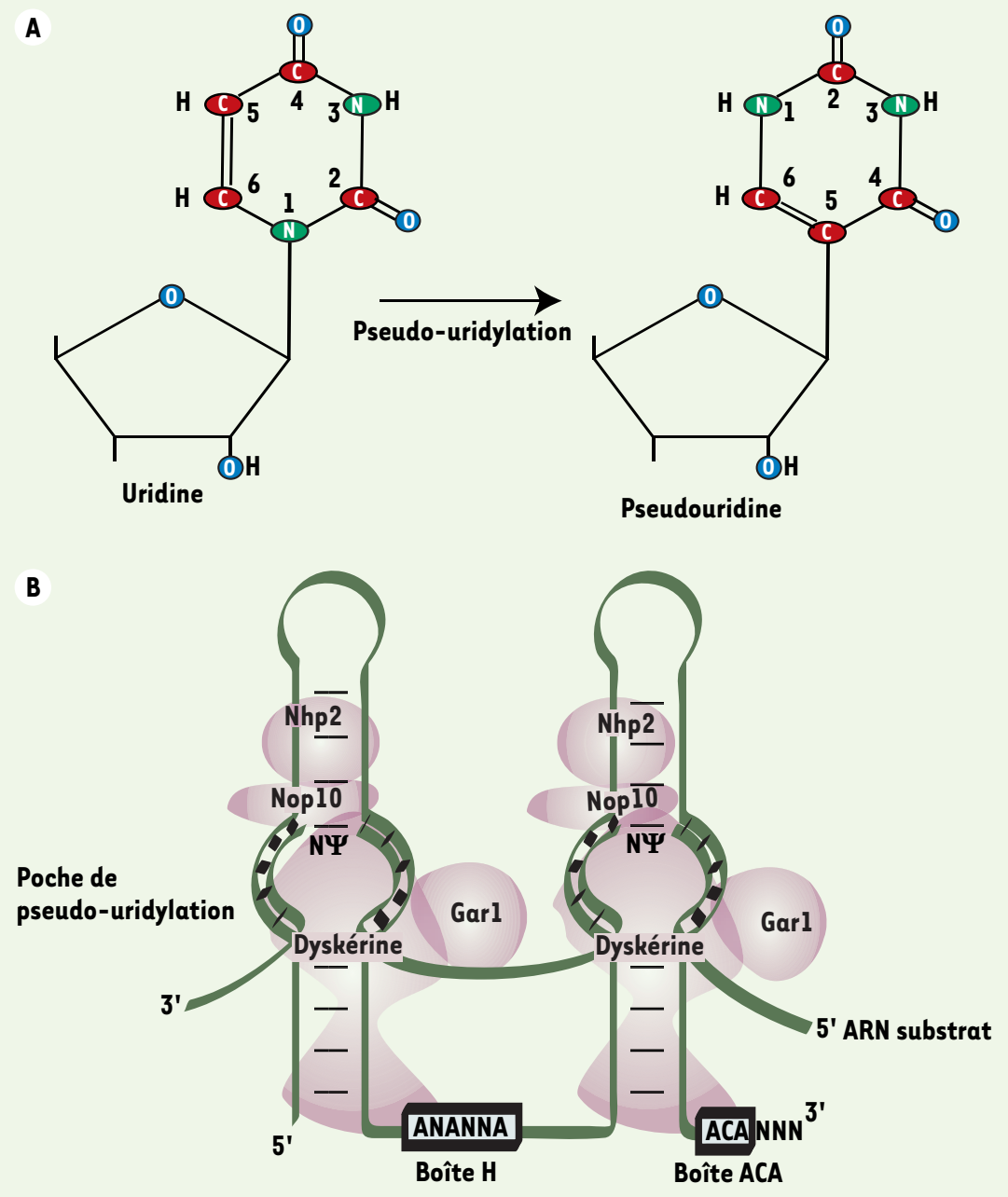

Figure 1. Fonction de la dyskérine. La protéine dyskérine peut catalyser des conversions d'uridine en pseudo-uridine quand elle est intégrée dans des particules H/ACA. A. Structures d'une uridine et d'une pseudo-uridine. B. Structure d'une particule H/ACA eucaryote. La position exacte des protéines au sein du complexe reste inconnue. gène codant I'ARN de la télomérase [41]. Les altérations de cet $A R N$ qui causent la $D C$ sont localisées avant tout dans la région dite pseudo-nœud qui jouxte la région matrice de l'ARN, et dans le domaine H/ACA (Figure 3). Certaines altérations du domaine H/ACA ont pour conséquence de déstabiliser l'ARN de la télomérase. Dans ce cas, les cellules souches des patients n'expriment que la forme normale de l'ARN de la télomérase, produite à partir de la copie saine du gène codant hTERC. La maladie est donc ici liée à un phénomène d'haplo-insuffisance. Les mutations affectant le domaine pseudo-nœud conduisent à la production de télomérase dont l'activité est fortement réduite, ce qui est responsable ici aussi d'une haplo-insuffisance [42-45].

Un autre gène qui semble responsable chez une famille de la forme autosomique dominante de la DC a été identifié en 2005 : il s'agit du gène codant la transcriptase inverse de la télomérase (hTERT) [46]. Dans ce cas également, la maladie est causée par un phénomène d'haplo-insuffisance. Des mutations dans le gène codant hTERT ont également été identifiées dans d'autres familles de patients atteints de DC, mais ces mutations ne ségrègent pas nécessairement avec la maladie [20]. Il est donc difficile de conclure quant à leur implication dans la maladie.

Dans les familles atteintes de la forme autosomique dominante de Ia DC, qu'elle soit due à des mutations dans le gène codant hTERC ou dans celui codant hTERT, on observe un phénomène d'anticipation génétique [44]. Ce terme désigne l'apparition plus précoce et l'accroissement de la sévérité des symptômes de la maladie au fur et à mesure des générations. Cela est corrélé avec un raccourcissement des télomères d'une génération à l'autre [47]. Des individus ayant hérité de la mutation et de télomères anormalement courts pourraient être incapables de restaurer une taille normale de leurs télomères en raison d'une activité de la télomérase insuffisante; dans cette hypothèse, plus les télomères des chromosomes hérités du parent porteur de la mutation sont courts, plus les symptômes de la maladie se déclareront tôt. 


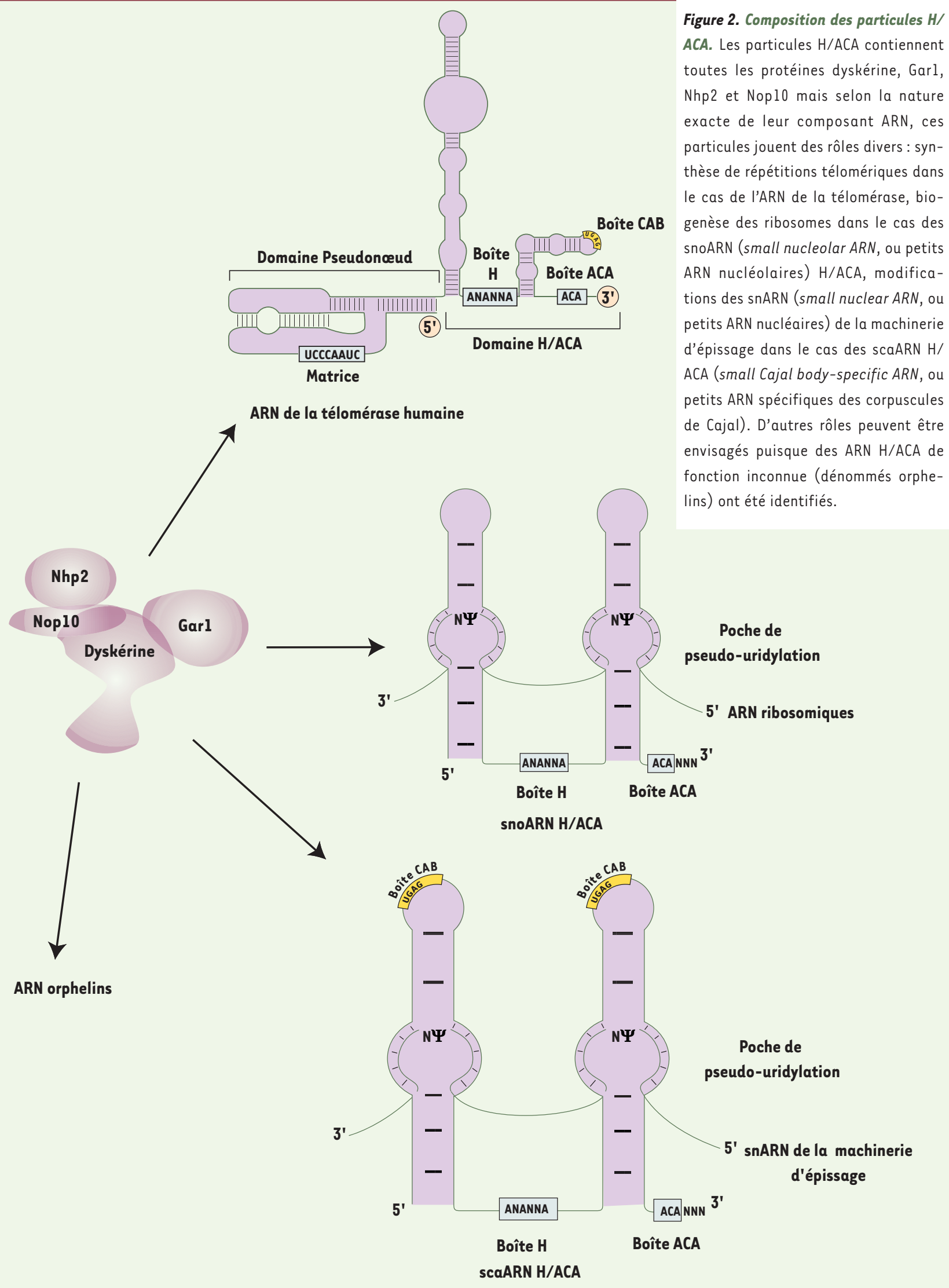


La forme autosomique récessive de la DC peut être causée par une mutation du gène codant la protéine Nop10

Une étude récente a conclu que plusieurs gènes étaient impliqués dans la forme autosomique récessive de la DC et a permis d'identifier l'un de ces gènes: il s'agit du gène codant la protéine Noplo [48]. Les cellules des individus malades présentent des télomères très courts et une forte diminution de la quantité de l'ARN de la télomérase. II est envisageable que la protéine Nop10 joue un rôle dans l'assemblage et/ou la stabilisation des RNP H/ACA et de la télomérase, mais cela reste à démontrer.

\section{La DC, un syndrome causé par une maintenance défectueuse des télomères}

La découverte de la responsabilité des mutations du gène codant dyskérine dans la forme de DC liée au chromosome $X$ suggéra initialement que la $D C$ résulte d'un défaut de synthèse des ribosomes [19]. La mise en évidence peu après que dyskérine fait partie, non seulement des RNP H/ACA participant à la synthèse des ribosomes, mais également de la télomerase conduisit à l'hypothèse que la DC résulte en fait d'une déficience d'activité télomerase conduisant à un défaut de maintien des télomères [31]. Cette dernière hypothèse

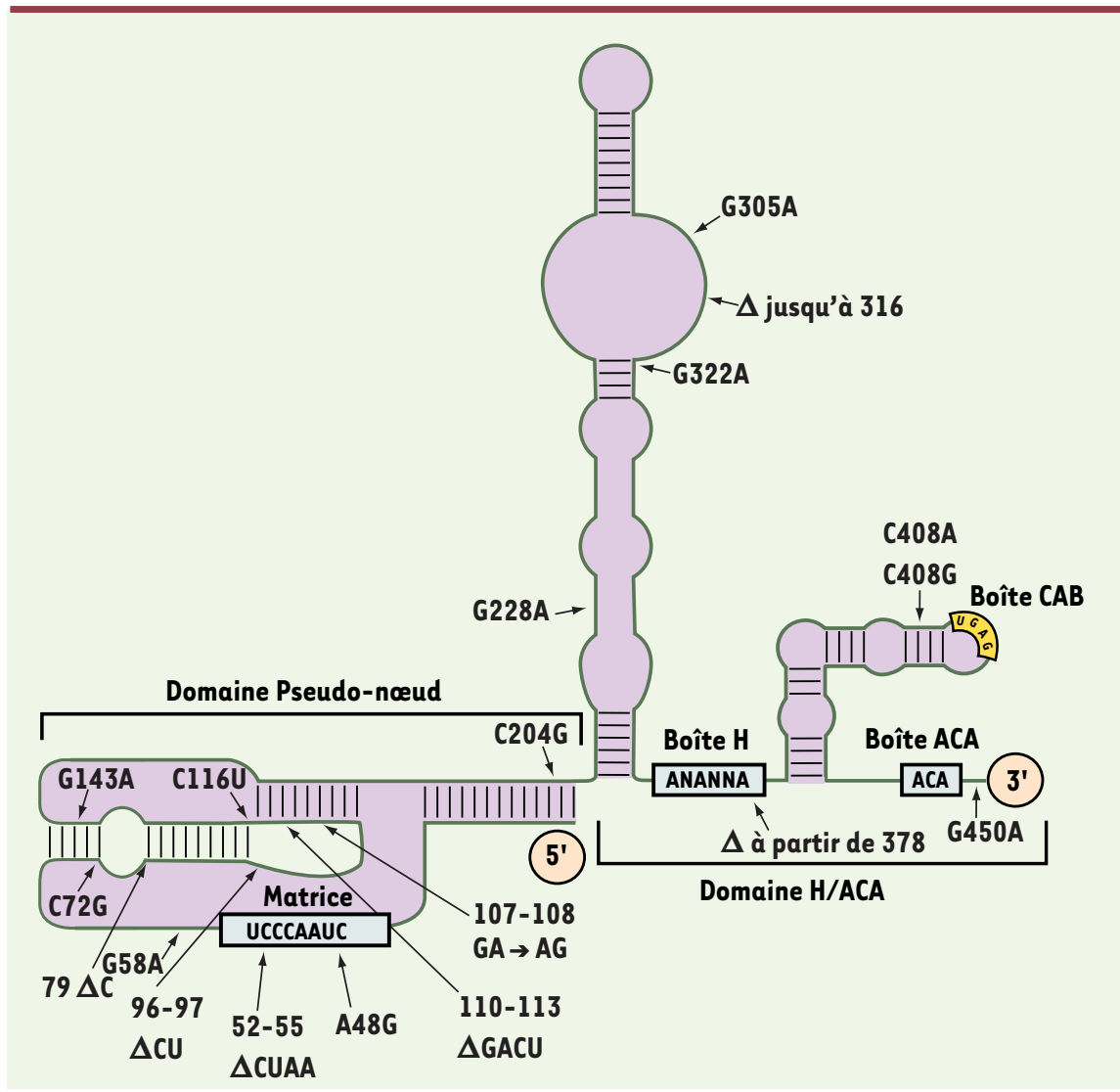

Figure 3. Structure secondaire de l'ARN de la télomérase humaine. Les mutations (remplacements ou suppressions de nucléotides) à l'origine de la forme autosomique dominante de la DC sont indiquées (d'après $[6,20,44]$ ). apparaît maintenant comme la plus valide, puisque la forme autosomique dominante de la DC résulte de mutations dans les gènes codant hTERC et hTERT, composants spécifiques de la télomérase qui ne sont a priori pas impliqués dans la synthèse des ribosomes. De plus, on note une corrélation entre la sévérité du phénotype clinique et le degré de réduction de la taille des télomères par rapport à des individus sains d'âge équivalent $[20,49]$.

Néanmoins, il est possible que les altérations de la dyskérine ou de Noplo responsables de cas de DC induisent également des défauts de synthèse des ribosomes, et donc de synthèse protéique, qui accroissent les symptômes. De fait, il a récemment été démontré que des cellules issues de patients atteints de DC due à des altérations de la dyskérine présentent un défaut très spécifique de la traduction initiée à partir de sites d'entrée interne des ribosomes sur les ARN messagers [50]. Ces défauts pourraient résulter en une baisse de facteurs anti-apoptotiques (qui contrecarrent la mort cellulaire) comme XIAP et $\mathrm{Bcl}-\mathrm{xL}$, et contribuer à la dégénérescence de la moelle osseuse. De manière intéressante, plusieurs autres atteintes constitutionnelles de la moelle osseuse (en particulier l'anémie de Diamond et Blackfan et le syndrome de Shwachman-Diamond) sont causées par des mutations de gènes codant des facteurs impliqués dans la biogenèse des ribosomes et la traduction (respectivement, des protéines ribosomiques et la protéine SBDS (Shwachman-Bodian-Diamond Syndrome protein) [51]. Cela étaye l'hypothèse selon laquelle des défauts de synthèse des ribosomes contribuent aux symptômes hématopoïétiques de la $D C$ liée à des mutations des gènes codant la dyskérine ou Noplo.

Il peut sembler paradoxal que les patients atteints de DC, un syndrome dû à un déficit d'activité télomérase, développent avec une fréquence élevée certains types de cancers, alors que la grande majorité des cancers humains sont associés à une réactivation de la télomérase $[52,61]$. Une des hypothèses plausibles est que la présence de télomères très courts dans certaines cellules induit une instabilité chromosomique propice à la transformation maligne. 


\section{Prise en charge et traitement}

La prise en charge des patients atteints de $D C$ est très lourde et suppose une approche multidisciplinaire. Elle est le plus souvent dominée par le traitement symptomatique de l'insuffisance médullaire et du déficit immunitaire qui va associer:

- Les androgènes, qui peuvent parfois améliorer le fonctionnement de la moelle pendant une période plus ou moins longue $[3,4]$.

- Un support transfusionnel, nécessaire quand les cytopénies sont sévères. - Des facteurs de croissance hématopoïétiques, G-CSF (granulocytecolony stimulating factor) en particulier, en traitement d'appoint d'une infection.

- Des traitements anti-infectieux curatifs et préventifs pour contrôler les infections favorisées par la neutropénie et l'immunosuppression.

Le seul traitement réellement curatif de l'insuffisance médullaire et du déficit immunitaire est l'allogreffe de cellules souches hématopoïétiques $[3,4]$. Malheureusement, les résultats de la greffe sont mauvais pour cette pathologie avec un risque de décès important lié à des complications pulmonaires et vasculaires survenant après la greffe [2, 53-55]. La forte incidence de complications pulmonaires pourrait être liée au fait que les patients atteints de DC ont intrinsèquement un risque d'évolution vers une fibrose pulmonaire. Pour cette raison il est logique d'exclure les agents associés à une toxicité pulmonaire (busulphan), de même que l'irradiation des poumons, lors du conditionnement des patients avant greffe. L'espoir actuel est lié à l'utilisation de conditionnements utilisant le cyclophosphamide et le sérum antilymphocytaire [56] ou la fludarabine [38,57] qui semblent donner des résultats encourageants.

- La thérapie génique reste un objectif théorique et il n'y a pas à ce jour de projet avancé en la matière.

\section{Perspectives}

La dyskératose congénitale reste une maladie trop peu connue, de diagnostic difficile, et souvent fatale. Pour améliorer le soutien aux

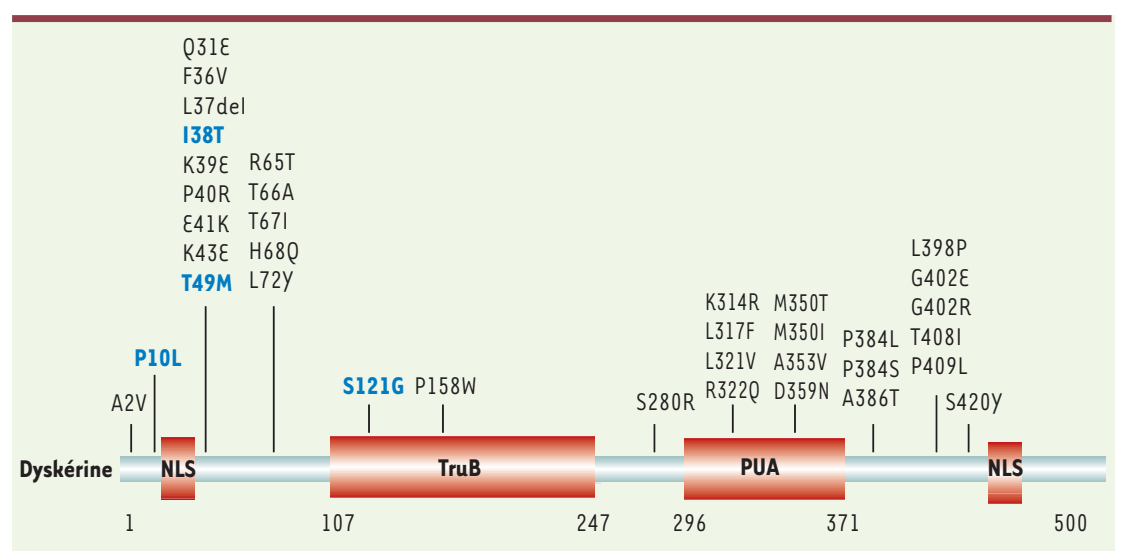

Figure 4. Structure primaire de la dyskérine. Les remplacements ou suppressions d'acides aminés à l'origine de la forme liée au sexe de la DC sont indiqués. En bleu : remplacements identifiés dans des cas de syndrome de HH (d'après [44]). familles et la prise en charge thérapeutique des malades $^{1}$, les actions suivantes doivent être poursuivies et développées:

- Permettre une meilleure diffusion des connaissances cliniques concernant cette maladie au sein de la communauté médicale et du public.

- Aider la recherche, afin de permettre tout à la fois l'identification de nouveaux gènes (actuellement la majorité des malades atteints de formes autosomiques de $D C$ ou de $H H$ ne présentent pas de mutation dans les gènes connus) et une meilleure compréhension de la physiopathologie de la maladie, en particulier l'identification de facteurs modificateurs du phénotype en fonction d'un même génotype.

- Améliorer les résultats des allogreffes de cellules souches hématopoïétiques par la mise au point de nouveaux conditionnements et la prévention, si possible, des complications tardives.

- Poursuivre la recherche en matière de thérapie génique. $\diamond$

\section{SUMMARY}

Dyskeratosis congenita, a disease caused by defective telomere maintenance Dyskeratosis congenita (DC), also called Zinsser-ColeEngman syndrome, is a rare, often fatal, inherited disease described for the first time at the dermatological level by Zinsser in 1906. It is a very polymorphous disease at the clinical level, with several modes of inheritance. Several clinical symptoms of the disease can appear after a latency period. These features render DC particularly difficult to diagnose. Mutations of several genes can cause DC, four of them having been identified so far. However, for a majority of patients, the affected gene has not been found. Remarkably, all identified genes ( $D K C 1$, $h T E R C, h T E R T$, and NOP10) encode components of telomerase, all required for telomere length maintenance. DC is thus a unique clinical model for the study of the roles of telomerase and telomeres. Moreover, proteins encoded by the DKCl and NOP10 genes are also components of so-called box H/ACA RNPs required for ribosome synthesis and pre-mRNA processing. Alterations of these processes could contribute to the symptoms of $D C$ patients carrying mutations in $D K C I$ or NOP10. $\diamond$

${ }^{1}$ Site de I'AFDC (Association française de la dyskératose congénitale) : www.afdc.asso.fr 


\section{REMERCIEMENTS}

Nous remercions David Villa pour l'aide dans la composition des figures et Anthony Henras et Michèle Caizergues-Ferrer pour leur relecture critique de l'article. Coralie Hoareau-Aveilla était financée par une bourse de l'Association pour la Recherche sur le Cancer (ARC). Les travaux de l'équipe d'yves Henry sont financés par le CNRS, I'Université Paul Sabatier, la Ligue Nationale contre le Cancer (équipe labellisée) et l'Agence Nationale de la Recherche.

\section{NOTE AJOUTÉદ AUX ÉPREUVES}

Après soumission de notre manuscrit, une communication faite à I'ASH (congrès de l'American Society of Hematology) a fait état de la découverte avec une fréquence élevée de mutations dans le gène TINF2 chez des patients atteints d'une forme de transmission autosomique dominante de $D C$ [58]. Ce gène code la protéine TIN2, une protéine faisant partie du «complexe de protection » (shelterin complex), qui se fixe à l'extrémité des télomères et joue un rôle crucial dans le maintien de leur intégrité [59]. Cette découverte confirme, s'il en était encore besoin, le lien entre $D C$ et défaut de maintien des télomères.

\section{RÉFÉRENCES}

1. Zinsser F. Atropha cutis reticularis cum pigmentatione, dystrophia ungiumet leukoplakia oris. Ikonogr Dermatol $1906 ; 5: 219-23$.

2. Knight $S$, Vulliamy $T$, Copplestone $A$, et al. Dyskeratosis congenita (DC) registry : identification of new features of DC. Br J Haematol 1998 ; 103 : 990-6.

3. Dokal I. Dyskeratosis congenita in all its forms. BrJ Haematol $2000 ; 110: 768-79$.

4. Dokal I. Fanconi's anaemia and related bone marrow failure syndromes. Br Med Bull 2006; 77-78: 37-53.

5. Mason PJ. Stem cells, telomerase and dyskeratosis congenita. Bioessays $2003 ; 25$ : 126-33.

6. Mason PJ, Wilson DB, Bessler M. Dyskeratosis congenita : a disease of dysfunctional telomere maintenance. Curr Mol Med $2005 ; 5$ : 159-70.

7. Dokal I, Vulliamy T. Dyskeratosis congenita : its link to telomerase and aplastic anaemia. Blood Rev 2003; 17 : 217-25.

8. Hoyeraal HM, Lamvik J, Moe PJ. Congenital hypoplastic thrombocytopenia and cerebral malformations in two brothers. Acta Paediatr Scand 1970; 59 : 185-91.

9. Fogarty PF, Yamaguchi $\mathrm{H}$, Wiestner $\mathrm{A}$, et al. Late presentation of dyskeratosis congenita as apparently acquired aplastic anaemia due to mutations in telomerase RNA. Lancet 2003 ; 362: 1628-30.

10. Vulliamy T, Marrone A, Dokal I, Mason PJ. Association between aplastic anaemia and mutations in telomerase RNA. Lancet 2002 ; 359 : 2168-70.

11. Vulliamy TJ, Walne A, Baskaradas A, et al. Mutations in the reverse transcriptase component of telomerase (TERT) in patients with bone marrow failure. Blood Cells Mol Dis 2005; $34: 257-63$.

12. Yamaguchi $H$, Baerlocher GM, Lansdorp $P$, et al. Mutations of the human telomerase RNA gene (TERC) in aplastic anemia and myelodysplastic syndrome. Blood 2003 ; 102 : 916-8.

13. Yamaguchi H, Calado RT, Ly H, et al. Mutations in TERT, the gene for telomerase reverse transcriptase, in aplastic anemia. N Engl J Med 2005 ; 352 : 1413-24.

14. Armanios MY, Chen JJ, Cogan JD, et al. Telomerase mutations in families with idiopathic pulmonary fibrosis. N EnglJ Med 2007 ; 356 : 1317-26.

15. Dokal I, Bungey J, Williamson P, et al. Dyskeratosis congenita fibroblasts are abnormal and have unbalanced chromosomal rearrangements. Blood $1992 ; 80: 3090-6$.

16. Vulliamy TJ, Knight SW, Mason PJ, Dokal I. Very short telomeres in the peripheral blood of patients with X-linked and autosomal dyskeratosis congenita. Blood Cells Mol Dis 2001 ; $27: 353-7$.

17. Drachtman RA, Alter BP. Dyskeratosis congenita : clinical and genetic heterogeneity. Report of a new case and review of the literature. Am J Pediatr Hematol Oncol 1992; 14 : 297-304.

18. Connor JM, Gatherer D, Gray FC, et al. Assignment of the gene for dyskeratosis congenita to Xq28. Hum Genet $1986 ; 72: 348-51$.

19. Heiss NS, Knight SW, Vulliamy TJ, et al. X-linked dyskeratosis congenita is caused by mutations in a highly conserved gene with putative nucleolar functions. Nat Genet 1998 ; $19: 32-8$.
20. Vulliamy TJ, Marrone A, Knight SW, et al. Mutations in dyskeratosis congenita : their impact on telomere length and the diversity of clinical presentation. Blood $2006 ; 107: 2680-5$.

21. Davis DR. Stabilization of RNA stacking by pseudouridine. Nucleic Acids Res $1995 ; 23: 5020-6$.

22. Meroueh M, Grohar PJ, Qiu J, et al. Unique structural and stabilizing roles for the individual pseudouridine residues in the 1920 region of Escherichia coli 23 rRNA. Nucleic Acids Res $2000 ; 28: 2075-83$.

23. Henras A, Henry Y, Bousquet-Antonelli C, et al. Nhp2p and Nop10p are essential for the function of H/ACA snoRNPs. EMBO J 1998 ; 17 : 7078-90.

24. Reichow SL, Hamma T, Ferré-D’Amaré AR, Varani G. The structure and function of small nucleolar ribonucleoproteins. Nucleic Acids Res 2007 ; $35: 1452-64$.

25. Meier UT. The many facets of H/ACA ribonucleoproteins. Chromosoma 2005 ; $114: 1-14$.

26. Henras AK, Dez C, Henry Y. RNA structure and function in C/D and H/ACA s(no)RNPs. Curr Opin Struct Biol $2004 ; 14: 335-43$.

27. Valadkhan $S$. snRNAs as the catalysts of pre-mRNA splicing. Curr Opin Chem Biol $2005 ; 9: 603-8$

28. Dönmez G, Hartmuth K, Lührmann R. Modified nucleotides at the 5 ' end of human U2 snRNA are required for spliceosomal $\varepsilon$-complex formation. RNA $2004 ; 10: 1925-33$.

29. Chen JL, Blasco MA, Greider CW. Secondary structure of vertebrate telomerase RNA. Cell $2000 ; 100: 503-14$.

30. Mitchell JR, Cheng J, Collins K. A box H/ACA small nucleolar RNA-like domain at the human telomerase RNA 3' end. Mol Cell Biol 1999; 19 : 567-76.

31. Mitchell JR, Wood $\varepsilon$, Collins K. A telomerase component is defective in the human disease dyskeratosis congenita. Nature $1999 ; 402: 551-5$.

32. Autexier $C$, Lue NF. The structure and function of telomerase reverse transcriptase. Annu Rev Biochem 2006 ; 75 : 493-517.

33. Moon IK, Jarstfer MB. The human telomere and its relationship to human disease, therapy, and tissue engineering. Front Biosci 2007 ; 12 : 4595-620.

34. Masutomi K, $Y_{u} \varepsilon y$, Khurts $S$, et al. Telomerase maintains telomere structure in normal human cells. Cell $2003 ; 114: 241-53$.

35. Pogačić V, Dragon F, Filipowicz W. Human H/ACA small nucleolar RNPs and telomerase share evolutionarily conserved proteins NHP2 and NOP10. Mol Cell Biol $2000 ; 20$ : 9028-40.

36. Li L, Ye K. Crystal structure of an H/ACA box ribonucleoprotein particle. Nature 2006 ; 443 : 302-7.

37. Normand C, Capeyrou R, Quevillon-Chéruel S, et al. Analysis of the binding of the $\mathrm{N}$-terminal conserved domain of yeast Cbf5p to a box H/ACA snoRNA. RNA $2006 ; 12$ : 1868-82.

38. Cossu F, Vulliamy TJ, Marrone A, et al. A novel DKCl mutation, severe combined immunodeficiency ( $\mathrm{T}+\mathrm{B}-\mathrm{NK}-\mathrm{SCID})$ and bone marrow transplantation in an infant with Hoyeraal-Hreidarsson syndrome. BrJ Haematol $2002 ; 119: 765-8$.

39. Knight SW, Heiss NS, Vulliamy TJ, et al. Unexplained aplastic anaemia, immunodeficiency, and cerebellar hypoplasia (Hoyeraal-Hreidarsson syndrome) due to mutations in the dyskeratosis congenita gene, $\mathrm{DKCl} . \mathrm{Br} J$ Haematol $1999 ; 107: 335-9$.

40. Sznajer Y, Baumann C, David A, et al. Further delineation of the congenital form of X-linked dyskeratosis congenita (Hoyeraal-Hreidarsson syndrome). Eur J Pediatr 2003 ; $162: 863-7$.

41. Vulliamy T, Marrone A, Goldman F, et al. The RNA component of telomerase is mutated in autosomal dominant dyskeratosis congenita. Nature 2001 ; $413: 432-5$.

42. Fu D, Collins K. Distinct biogenesis pathways for human telomerase RNA and H/ACA small nucleolar RNAs. Mol Cell 2003; 11 : 1361-72.

43. Marrone A, Stevens D, Vulliamy T, et al. Heterozygous telomerase RNA mutations found in dyskeratosis congenita and aplastic anemia reduce telomerase activity via haploinsufficiency. Blood 2004 ; 104 : 3936-42.

44. Marrone A, Walne A, Dokal I. Dyskeratosis congenita : telomerase, telomeres and anticipation. Curr Opin Genet Dev $2005 ; 15: 249-57$.

45. Cerone MA, Ward RJ, Londono-Vallejo JA, Autexier C. Telomerase RNA mutated in autosomal dyskeratosis congenita reconstitutes a weakly active telomerase enzyme defective in telomere elongation. Cell Cycle 2005 ; 4: 585-9.

46. Armanios M, Chen JL, Chang YP, et al. Haploinsufficiency of telomerase reverse transcriptase leads to anticipation in autosomal dominant dyskeratosis congenita. Proc Natl Acad Sci USA 2005 ; 102 : 15960-4.

47. Vulliamy T, Marrone A, Szydlo R, et al. Disease anticipation is associated with progressive telomere shortening in families with dyskeratosis congenita due to mutations in TERC. Nat Genet $2004 ; 36: 447-9$. 


\section{Association Française de la Dyskératose Congénitale (AFDC)}

Pour aider les patients et leurs familles, mais aussi les médecins à diagnostiquer la Dyskératose Congénitale (DC), l'Association Française de la Dyskératose Congénitale (AFDC) a été créée en avril 2007, s'engageant à œuvrer pour une meilleure connaissance de la DC et de ses formes atypiques et à soutenir la recherche fondamentale et clinique sur cette pathologie. Structurer et renforcer les moyens de l'association, améliorer l'accès à l'information, au diagnostic et aux soins, et favoriser la recherche médicale constituent les trois axes prioritaires de I'AFDC. Elle est assistée d'un conseil médico-scientifique, composé de Madame Corinne Pondarré, médecin hématologue, service d'immunohématologie pédiatrique, Hôpital Debrousse à Lyon et de Messieurs

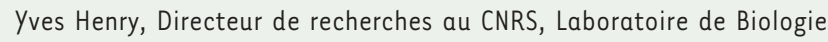
Moléculaire Eucaryote à Toulouse, Tamas Kiss, Directeur de recherches à I'Inserm, Laboratoire de Biologie Moléculaire Eucaryote à Toulouse et Thierry Leblanc, médecin hématologue, du Centre de Référence sur les Aplasies Médullaires acquises et constitutionnelles, hôpital SaintLouis à Paris. Les membres du Conseil administratif de l'AFDC assurent diverses responsabilités d'orientation générale, de gestion financière et de communication.

Coordonnées de l'AFDC : 10 rue des Aires, 34980 Montferrier-sur-Lez, France.

Tél : 0620925992

Site internet : http://www.afdc.asso.fr

Présidente : Mme Sonia Schmidtbauer

sonia.schmidtbauer@afdc.asso.fr
48. Walne AJ, Vulliamy T, Marrone A, et al. Genetic heterogeneity in autosomal recessive dyskeratosis congenita with one subtype due to mutations in the telomerase-associated protein NOP10. Hum Mol Genet $2007 ; 16: 1619-29$.

49. Alter BP, Baerlocher GM, Savage SA, et al. Very short telomere length by flow fluorescence in situ hybridization identifies patients with dyskeratosis congenita. Blood 2007 ; 110 : 1439-47.

50. Yoon A, Peng G, Brandenburger Y, et al. Impaired control of IRESmediated translation in $\mathrm{X}$-linked dyskeratosis congenita. Science 2006 ; $312: 902-6$.

51. Liu JM, Ellis SR. Ribosomes and marrow failure : coincidental association or molecular paradigm? Blood $2006 ; 107: 4583-8$.

52. Kim NW, Piatyszek MA, Prowse KR, et al. Specific association of human telomerase activity with immortal cells and cancer. Science 1994 ; 266: 2011-5.

53. Langston AA, Sanders JE, Deeg HJ, et al. Allogeneic marrow transplantation for aplastic anaemia associated with dyskeratosis congenita. BrJ Haematol $1996 ; 92$ : 758-65.

54. Rocha V, Devergie A, Socie G, et al. Unusual complications after bone marrow transplantation for dyskeratosis congenita. Br J Haematol 1998 ; $103: 243-8$.

55. Yabe $M$, Yabe $H$, Hattori $K$, et al. Fatal interstitial pulmonary disease in a patient with dyskeratosis congenita after allogeneic bone marrow transplantation. Bone Marrow Transplant 1997 ; 19 : 389-92.

56. Lau YL, Ha SY, Chan CF, et al. Bone marrow transplant for dyskeratosis congenita. BrJ Haematol 1999; 105 : 571.

57. Nobili B, Rossi G, De Stefano P, et al. Successful umbilical cord blood transplantation in a child with dyskeratosis congenita after a fludarabine-based reduced-intensity conditioning regimen. BrJ Haematol 2002 ; 119 : 573-4.

58. Savage SA, Giri N, Baerlocher GM, et al. TINF2, a component of the shelterin telomere protection complex, is mutated in Dyskeratosis congenita. Am J Hum Genet $2008 ; 82$ : $501-9$.

59. De Lange T. Shelterin : the protein complex that shapes and safeguards human telomeres. Genes Dev $2005 ; 19: 2100-10$.

60. Rufer N, Nabholz M. Télomérase, élixir de jouvence des cellules humaines? Med Sci (Paris) $2003 ; 19: 345-50$.

61. Brunori $M$, Gilson $\varepsilon$. Télomère et cancer : quoi de plus à la fin ? Med $\mathrm{Sci}$ (Paris) $2005 ; 21: 37-42$.

62. Londono-Vallejo A, Lenain C, Gilson $\varepsilon$. Cibles les télomères pour forcer les cellules cancéreuses à rentrer en sénescence. Med Sci (Paris) 2008 ; $24: 383-9$.

63. Gilgenkrantz H. La longévité : un héritage paternel. Med Sci (Paris) 2007 ; $23: 812$.

\title{
Y. Henry
}

\begin{abstract}
23:812.
\end{abstract}

\section{TIRÉS À PART}

Y. Henry

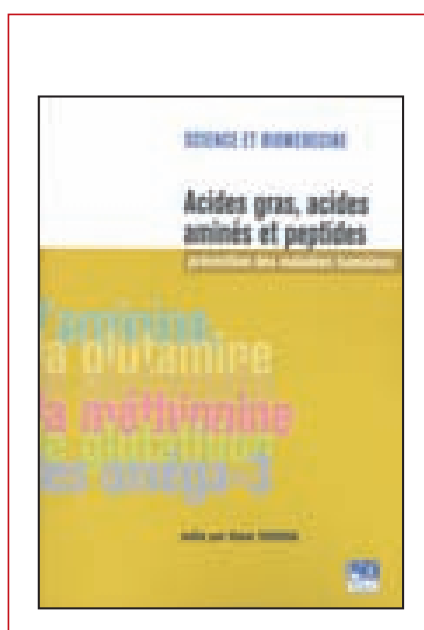

\section{Bon de commande}

À retourner à EDK, 2, rue Troyon - 92316 Sèvres Cedex

Tél. : 0155641393 - Fax : 0155641394 - E-mail : edk@edk.fr

NOM : Prénom :

Adresse :

Code postal :

Ville :

Pays :

Fonction :

Je souhaite recevoir l'ouvrage Acides gras, acides aminés et peptides : $12 €+3 €$ de port $=\mathbf{1 5} €$ TTC

en ................. exemplaire, soit un total de ......................................... €

Par chèque, à l'ordre de $\mathbf{E} \mathbf{D} \mathbf{K}$

Par carte bancaire : Visa $\quad$ Eurocard/Mastercard

Carte $\mathrm{n}^{\circ}$

- Signature :

ISBN : 2-84254-108-1 80 pages

Date d'expiration :

$\mathrm{N}^{\circ}$ de contrôle au dos de la carte : 